The guidance relating to consent to treatment is of vital importance for all medical practitioners (not only in relation to mental disorder) and provides a basis for discussion. It would be appropriate in a discussion document but its relevance here is questionable in that the law is not unequivocal in this area.

The extension of rules to research has been discussed eisewhere, ${ }^{8}$ and is clearly of vital concern to many workers. Here, as elsewhere, the full implications of the recommendations do not appear to have been considered.

There are relatively few practice directions appropriate to the Code and these have to be distinguished with difficulty from the detailed comment, explanation, observations and other matter in the draft.

\section{Discussion}

The draft Code of Practice has been circulated widely to the representatives of professions, to the Royal Colleges, Regional and District Health Authorities and Community Health Councils. It has serious implications for all the professions concerned with the care of the mentally disordered and for the future treatment and management of patients. It is of equal concern to all members of the medical profession, not only as the first code to influence medical practice but also because its ramifications, in draft, will concern all doctors, not only psychiatrists.
It is important that all those consulted should respond only after careful scrutiny of the draft to ensure that the final document is concise, pertinent, relevant, simple to understand, advances the rights and care of the patient and respects the obligations and professional judgement of practitioners. It is important to be aware of its possible future legal status.

The gems of practice guidance must be filleted out from all the rest and these may then form the basis for a simple and useful Code.

REFERENCES

${ }^{1}$ Mental Health Act 1983. London: HMSO.

${ }^{2}$ Grout, T. J. (1984) Public Law. London: McDonald \& Evans.

${ }^{3}$ House of LoRds Weekly Hansard (1986) No. 1310. Cols. 1076-1078. 15 January 1986.

${ }^{4}$ House of Lords Weekly Hansard (1986) No. 1310. Col. 1086. 15 January 1986.

${ }^{5}$ House of Lords Weekly Hansard (1986) No. 1310. Col. 1083. 15 January 1986.

'Official Report of the Special Standing Committee on the Mental Health (Amendment) Bill (Lords) Part II (1982) 25 May-29 June 1982. London: HMSO.

${ }^{7}$ House of Lords Weekly Hansard (1986) No. 1310. Col. 1079. 15 January 1986.

${ }^{8}$ Kendell. R. The Mental Health Act Commission's 'Guidelines': a further threat to psychiatric research to appear in May 1986 Bulletin of the Royal College of Psychiatrists.

\title{
The Sheffield Cognitive Psychotherapy Training Course
}

N. D. MacaskILL, Consultant with a Special Interest in Psychotherapy, Whiteley Wood Clinic, Sheffield

This paper describes a basic training course in cognitive therapy for trainee psychiatrists on the Sheffield rotational scheme. Cognitive therapy, as represented by the work of Beck and Ellis, has emerged over the past decade as a potent therapeutic tool, particularly in the treatment of depression. It is a structured, directive, short-term therapy aimed at eliciting and modifying the dysfunctional cognitions which are hypothesised to play a central role in initiating and maintaining emotional disorders.

To date, guidelines for psychotherapy training for psychiatrists issued by the Royal Medico-Psychological Association British Journal of Psychiatry (1971) ${ }^{1}$ and personal recommendations, e.g. Brandon $(1982)^{2}$, have omitted any reference to training in cognitive therapy. However laudable these basic guidelines are, it can be argued that they are significantly under-inclusive. Training in cognitive therapy is, as a result, not likely to receive the emphasis and support it deserves and in future years trainee psychiatrists who have had no training in cognitive therapy could be seen as lacking a genuinely comprehensive psychotherapeutic approach to the psychological management of their patients. It was this point of view that led to the development of the basic training course in cognitive psychotherapy described below.

Trainees in the Sheffield rotational scheme all receive regular supervision in dynamic psychotherapy throughout their training, in line with the Royal College 1971 guidelines, and in addition have the opportunity for supervision in behaviour therapy, group therapy and psychological management of marital and sexual problems. The cognitive therapy group was designed to build on the general psychotherapeutic skills acquired by trainees in dynamic supervision. Trainees are required to have at least six months prior general psychotherapeutic supervision, as a good grounding in relationship-building and interviewing skills are essential if the confronting and directive techniques of cognitive therapy are to be optimally effective.

\section{Aims}

The course firstly aims to provide a systematic presentation and a study of Beck's cognitive therapy. Beck's work is used as a basis for the course because it is clearly described in numerous publications, backed by detailed treatment manuals and audio-visual materials of real and simulated 
cognitive therapy and utilises a wide variety of cognitive and behavioural methods in the standard treatment package. Building on Beck's work, the course introduces trainees to the work of Ellis and rational-emotive psychotherapy. This cognitive therapy complements Beck's approach in altering core belief systems believed to be important in predisposing patients to emotional disorders.

Finally, the course encourages trainees in the regular use of objective measures of psychopathology and change, systematic assessment and planning in therapy and a critical assessment of the research literature and its relevance to clinical practice. This empirical approach to psychotherapeutic practice is explicit in Beck's work and it is hoped provides trainees with a valuable model.

\section{Methods}

Trainees meet weekly for one and a half hours for 20 weeks. Each weekly session is divided into an introductory discussion of theoretical and/or practical aspects of cognitive therapy followed by supervision of trainees' cases. Supervision initially uses process notes, but in the latter half of the course, it utilises audio-taped review of sessions. Trainees are encouraged to supervise all their own sessions systematically. A detailed therapy session rating manual and scale (courtesy of Centre for Cognitive Therapy) enables trainees to maximise opportunities for supervision as only a small proportion of each trainee's tapes can be reviewed in the weekly group supervision sessions. The scores on the therapy rating scale are used as indices of therapists' competence and provide trainee and supervisor with systematic information to focus further learning. Trainees' tapes can also be submitted to the course supervisor for more detailed feedback throughout the course, as well as listened to in the weekly sessions. Tapes of real and simulated sessions of cognitive therapy conducted by Beck and others are also used in the early sessions. These tapes provide a valuable supplement to the reading material, bring cognitive therapy to life and substantially enhance learning. Tapes of sessions by a variety of experts in cognitive therapy can be reviewed by trainees throughout the course at their own leisure.

After four weeks of the course trainees may take on selected out-patients. Over the time scale of the course trainees are expected to treat three to four patients mainly with depressive disorders, but also including those with anxiety problems, phobic states and relationship problems.

At the end of the course each trainee is expected to submit a protocol of a patient he/she has treated for a minimum of ten sessions. The protocol is expected to demonstrate a basic knowledge of cognitive theory and a practical application of cognitive-change techniques.

\section{Evaluation}

The first cohort of five trainees has completed the course and an evaluation can now be made. By the end of the course trainees were generally functioning at a satisfactory level in terms of structuring sessions, planning homework, eliciting cognitions and utilising appropriate cognitivechange techniques. The review of tape sessions indicated, however, a need for further supervision to improve expertise in the application of cognitive-change techniques and in planning overall therapeutic strategy. In the initial stages of the course trainees experienced a 'de-skilling phase' arising out of their efforts to shift from a reflective, analytic style to an active directive style of working. This phase seemed to last about six weeks on average.

The five trainees were all satisfied with and enjoyed the course, indicated that it had led to a generalisation of cognitive concepts and techniques to their psychotherapeutic work and as a result of the course they all expressed an interest in further training in cognitive therapy. All expressed a view that self-supervision and supervisor review of tapes had been very useful. There was general agreement that a more extended opportunity to read relevant material before the course would have been helpful. Following the course trainees decided to meet regularly for peer-group supervision. Fourteen patients were treated during the course, ten improved and one patient dropped out. A few patients improved sufficiently to terminate therapy by mutual agreement in three to six sessions though a substantial proportion of patients were still in treatment at the end of the course. Patient problems treated include depression, anxiety and phobias. Trainees found Ellis' rational-emotive therapy of particular interest and relevance to their cognitive work. This seemed to reflect a predisposition to work towards personality change rather than restricting themselves to teaching patients methods of controlling and coping with their automatic dysfunctional thoughts. It was also evident that trainees made frequent use of objective measures of change and psychopathology in their work by the end of the course.

Interest in training in cognitive therapy is increasing, for example, Scott et al (1985) ${ }^{3}$ have described a workshop and peer supervision training scheme in Newcastle. The present course offers an alternative model and has shown that the relatively brief training can achieve useful levels of skills in cognitive therapy. Further modification of the course and evaluation of the trainees' progress over the next twelve months will be undertaken to determine the optimum structure required to enable trainees to reach a level where they can function independently as cognitive therapists.

REFERENCES

${ }^{2}$ Royal Medico-Psychological Association (1971) Memorandum on future patterns of care for the mentally subnormal. British Journal of Psychiatry, 119, 95-99.

${ }^{2}$ Brandon, S. (1982) Training in psychotherapy-A personal view. Bulletin of Royal College of Psychiatrists, 6, 120.

${ }^{3}$ SCOTt, J., BARKER, W. A. \& WILLIAMS, J. G. (1985) The teaching of cognitive therapy in Newcastle. Bulletin of Royal College of Psychiatrists, 9, 33-34. 